\title{
Publisher's Note: Measurement of the antineutrino to neutrino charged-current interaction cross section ratio in MINERvA [Phys. Rev. D 95, 072009 (2017)]
}

\author{
L. Ren et al. \\ (MINERvA Collaboration)
}

(a) (Received 5 January 2018; published 30 January 2018)

DOI: 10.1103/PhysRevD.97.019902

This paper was published online on 14 April 2017 with a typographical error in Eq. (11). Equation (11) should read as

$$
R_{C C}(E)=\frac{\sigma_{C C}^{\bar{\nu}}(E)}{\sigma_{C C}^{\nu}(E)}=\frac{\mathcal{R}^{\bar{\nu}}}{\mathcal{R}^{\nu}}\left(\frac{A_{C C}^{\bar{\nu}, \mathrm{KIN}}(E) \times S^{\bar{\nu}}\left(\nu_{0}, E\right) \times H^{\nu}\left(\nu_{0}\right)}{A_{C C}^{\nu, \mathrm{KIN}}(E) \times S^{\nu}\left(\nu_{0}, E\right) \times H^{\bar{\nu}}\left(\nu_{0}\right)}\right) .
$$

The equation has been corrected as of 10 January 2018. The equation is incorrect in the printed version of the journal. 